\title{
Lumen
}

Selected Proceedings from the Canadian Society for Eighteenth-Century Studies

\section{Le spectacle et le récit. Petite poétique du roman asmodéen}

\section{Ugo Dionne}

Volume 22, 2003

URI : https://id.erudit.org/iderudit/1012263ar

DOI : https://doi.org/10.7202/1012263ar

Aller au sommaire du numéro

Éditeur(s)

Canadian Society for Eighteenth-Century Studies / Société canadienne d'étude du dix-huitième siècle

ISSN

1209-3696 (imprimé)

1927-8284 (numérique)

Découvrir la revue

Citer cet article

Dionne, U. (2003). Le spectacle et le récit. Petite poétique du roman asmodéen.

Lumen, 22, 135-148. https://doi.org/10.7202/1012263ar

Copyright (C Canadian Society for Eighteenth-Century Studies / Sociéte canadienne d'étude du dix-huitième siècle, 2003
Ce document est protégé par la loi sur le droit d'auteur. L'utilisation des services d'Érudit (y compris la reproduction) est assujettie à sa politique d'utilisation que vous pouvez consulter en ligne.

https://apropos.erudit.org/fr/usagers/politique-dutilisation/ 


\section{Le spectacle et le récit. Petite poétique du roman asmodéen}

On constate, chez les lecteurs français des $\mathrm{XVII}^{\mathrm{e}}$ et $\mathrm{XVIII}{ }^{\mathrm{e}}$ siècles, une véritable avidité narrative, une satisfaction spécifique, liée à une prolifération apparemment gratuite des récits insérés, successifs ou imbriqués. La question de la pertinence dramatique ou herméneutique d'une «histoire» reste ici tout à fait secondaire: chaque nouveau récit, chaque nouveau «départ» narratif, est a priori désirable, donc a priori justifié, à défaut de nous sembler toujours absolument motivé ${ }^{1}$. Cette narratophilie éclaire un certain nombre de phénomènes, qui pourraient autrement

1 Ce phénomène a bien sûr déjà été signalé par la critique, fût-ce de manière incidente, à la faveur d'un commentaire adventice ou d'une digression. Vivienne Mylne précise ainsi que le principal intérêt du roman français du XVIII ${ }^{\mathrm{e}}$ siècle réside, pour ses lecteurs, "in their story-line, in the adventures, in the element of what-happens-next. At this level, any story with lively events is a good thing; and the more stories a novel contains, the better it will be. [...] the multiplicity of separate tales, the speed of events, the way that novelists press on to the next adventure, all seem to bear out the notion that the story-line was the prime object of interest for writer and reader alike» (The Eighteenth-Century French Novel. Techniques of Illusion, Cambridge, Cambridge University Press, 1982, $2^{\mathrm{e}}$ édition, p. 61-62; nos italiques). Henri Coulet (Marivaux romancier. Essai sur l'esprit et le cœur dans les romans de Marivaux, Paris, Armand Colin, coll. «Publications de la Sorbonne», 1975, p. 412-413) et Annie Rivara (les Sours de Marianne: suites, imitations, variations: 1731-1746, Studies on Voltaire and the Eighteenth Century, 1991, p. 27-36) ont par ailleurs tous deux remarqué le mode de lecture et de réception "ponctuel" qu'entraîne, dans les années 1730 et 1740 , la pratique de la publication "périodique» du roman, inaugurée par Marivaux. Or cette lecture parcellaire - qui considère chaque livraison du roman comme une unité en soi, appréciable immédiatement, selon ses termes propres, sans que soit sans cesse évoquée la perspective d'une œuvre "totale», éventuellement achevée, que l'on peut bien sûr continuer à espérer, mais sur laquelle l'expérience interdit de trop compter - nous semble elle aussi informée par la dynamique narratophilique de l'Ancien Régime. Cette dynamique, à laquelle on n'a pas encore accordé toute l'attention qu'elle mérite, explique autant la relative nonchalance du public de l'Ancien Régime devant l'inachèvement $d^{\prime}$ un roman que son consentement tacite aux suites (autographes ou apocryphes), aux continuations, aux reprises et aux «réouvertures» dont l'ouvrage «clos» fait lui-même fréquemment l'objet. 
surprendre l'historien de la littérature. C'est à elle que nous tendrions par exemple à attribuer, au moins en partie, le succès phénoménal du roman héroïque des années 1640-1660: le gigantisme de Cléopâtre ou de Clélie, inlassablement fustigé dès la génération suivante, ne correspondrait en somme qu'à l'espace, à la superficie nécessaires pour loger les péripéties foisonnantes, les personnages multiples, la matière romanesque proliférante que goûtait le lecteur préclassique. C'est encore à ce désir, à cette appétence du récit, que nous suggérons d'imputer le développement, au XVIII ${ }^{\mathrm{e}}$ siècle - à côté ou en sus des recueils parataxiques, des collections de nouvelles ou des structures à tiroirs héritées du roman baroque -, de formules romanesques dont la seule fonction semble être de permettre et de favoriser l'amoncellement narratif.

On pense ici, par exemple, au roman mangoguléen, bâti sur le modèle des Bijoux indiscrets de Diderot, dans lequel un personnage se voit doté d'un pouvoir magique (représenté, ou non, par un talisman ou un artefact merveilleux), lequel lui permet de «faire parler» un certain type d'objet, ainsi élevé à la dignité d'un narrateur métadiégétique. Les objets en question — sexes féminins, miroirs, bois de lit — renvoient à une intimité, à une vie secrète et privée, dont ils sont les seuls témoins et qui se trouve ainsi miraculeusement (et fort impudiquement) dévoilée ${ }^{2}$. On peut aussi évoquer une autre formule, anthropomorphique,

2 Outre les Bijoux indiscrets, la formule informe un certain nombre d'ouvrages du XVIII ${ }^{\mathrm{e}}$ siècle. Diderot lui-même a sans doute développé le thème traditionnel des sexes parlants, déjà recensé chez La Fontaine ou dans le fabliau, à la suite du Nocrion de Bernis (1747). Dans l'Optique, ou le Chinois à Memphis de Guérineau de Saint-Péravi (1763), c'est une lorgnette (et non plus un anneau, comme chez Mangogul), qui permet au narrateur d'observer le "fond des âmes»; le dispositif ainsi mis en place, susceptible de multiples développements, se replie cependant immédiatement sur deux histoires, celles de Neyda et d'Erzecan, qui occupent respectivement la première et la seconde partie du roman. Une semblable restriction de la formule peut être observée dans le Miroir des princesses orientales de Mme Fagnan (1755), qui, à partir d'un scénario propice à la multiplication narrative - un miroir indiscret révèle les pensées de ceux et de celles qui s'y contemplent -, ne développe guère que deux «nouvelles», attribuant un volume à chacune d'elles. Le schéma diderotien est toutefois repris systématiquement dans le Lit de noce, un roman anonyme de 1791 dont le héros, à la suite de diverses macérations et fustigations alchimiques, a acquis le pouvoir de converser avec les bois de lit. Les segments narratifs de l'ouvrage sont dès lors centrés sur les différents exercices, les mises à l'épreuve de ce pouvoir, comme le signalent les intertitres en inventaire («Première nuit», "Seconde nuit»...), rappelant directement ceux des Bijoux indiscrets. La parenté est d'autant plus probante, en l'espèce, que les «nuits» recensées sont uniquement celles où les facultés merveilleuses du héros-narrateur font l'objet d'un essai. 
dans laquelle un objet devient lui-même narrateur (ou le narrateur un objet...) et procède à une récapitulation de ses précédents propriétaires. On reconnaît le modèle du Sopha de Crébillon (1742), qui donnera lieu, tout au long du siècle, à des variations diversement obscènes ${ }^{3}$. Le roman picaresque, tel qu'on le pratique en France à partir des dernières années du $\mathrm{XVII}{ }^{\mathrm{e}}$ siècle, peut lui-même être perçu comme une structure d'accueil, comme un véritable roman spicilège. Le récit $\mathrm{du}$ pícaro se caractérise en effet par deux modes d'articulation contradictoires, tous deux susceptibles de (re)lancer la machine narrative. Il est d'abord cumulatif, donc linéaire: relation d'une vie errante, dominée par le hasard, dont elle épouse la forme aléatoire, $l^{\prime}$ «intrigue» picaresque juxtapose des épisodes indépendants; un maître, un état succèdent à un autre, jusqu'au bout du chemin, jusqu'au bout du roman. Le récit n'est pas unifié par la liaison causale des péripéties, mais par le motif de la route, elle-même pure succession, lieu toujours (et jamais) semblable à lui-même, où s'effectue un voyage dont «ni l'itinéraire ni le but ne sont clairement définis ${ }^{4} »$. L'intrigue picaresque, d'autre part, est digressive: aux lacets et aux méandres de la route correspondent autant de rencontres, tantôt avec de nouveaux personnages, tantôt avec des figures déjà croisées, que leurs propres parcours

3 Dans le Pythagore moderne (anonyme, 1762), le héros transformé en godemiché fait la joie des pensionnaires d'un couvent, avant d'en sortir et de pénétrer dans le monde. Le même type d'indiscrétion, dans un registre (à peine) moins scabreux, est fourni par la Jarretière de Teubern, traduite par Mme de Rome en 1769. Dans le Canapé couleur de feu de Fougeret de Montbron (1741), le narrateur est miraculeusement mué en divan, témoin et chroniqueur des (d)ébats qu'il soutient et favorise; c'est le schéma narratif qu'emploie aussi Crébillon dans le Sopha, alors que, invité par le sultan Schah-Baham à produire une histoire, le courtisan Amanzéi lui raconte comment, puni par Brahma pour ses mœurs dissipées, il a été condamné à transmigrer d'un sofa à un autre, dans les petites et les grandes maisons d' «Agra", jusqu'à ce que deux amants sincères se consacrent sur lui leurs pucelages. Plusieurs des applications de la formule sont adaptées de l'étranger, notamment de l'Anglais; ce picaresque animiste semble constituer, dans la littérature anglaise du XVIII ${ }^{\mathrm{e}}$ siècle, un genre à part entière, dont certaines réalisations ont traversé la Manche. Le Pompey the Little de Coventry relate les pérégrinations d'un petit chien; il est traduit par Toussaint en 1752, puis par Briel en 1784. Dans le Chrysal de Johnston, une pièce de monnaie se remémore, à l'intention de son nouveau possesseur, la suite des mains par lesquelles elle a transité; le texte est traduit en deux livraisons, de 1767 et 1769 , et sa prémisse sera adaptée par Caraccioli dans un petit opuscule de 1778, l'Écu de six francs. Enfin, dans les Aventures d'un habit noir, traduit par Girard-Raigné en 1790, «Black», le vieux manteau du titre, décline pour «White», un jeune vêtement présomptueux, la liste de ses anciens propriétaires.

4 Francis Assaf, LeSage et le picaresque, Paris, Nizet, 1983, p. 10. 
ont transformé, et qui s'élucident par la narration ${ }^{5}$. La prolifération du récit picaresque prend donc une double forme. Défilent, d'une part, les aventures sans cesse recommencées du «héros», séquence de vignettes et d'épisodes fermés sur eux-mêmes, qui entraînent une multiplication syntaxique de la matière narrative; s'imposent, d'autre part, les histoires insérées, généralement des récits rétrospectifs, mais parfois aussi des fictions et des «nouvelles» indépendantes, que lisent ou se récitent les personnages.

C'est toutefois à une autre de ces formules d'inspiration «narratophilique» que nous consacrerons ces pages: celle du roman asmodéen, en référence et en hommage au Diable boiteux de Le Sage, qui lui sert à la fois de paradigme, de prototype et de principale illustration. Pour peu qu'on y prête attention, le roman devient le point d'origine d'une filière, d'un «courant» traversant tout le XVIII ${ }^{e}$ siècle, et dont le caractère parfois extrêmement dérivé n'empêche pas l'identification d'un modèle ou d'un canevas communs. Nous tenterons d'abord de dégager la structure archétypale de ce roman asmodéen, en examinant méthodiquement son texte-source et en classant les différentes cellules (méta-)narratives qui le composent. Nous recenserons ensuite quelques-uns des détournements, quelques-unes des transformations et des déviations plus ou moins prononcées auxquels la formule a donné lieu; nous nous intéresserons plus particulièrement - en accord avec le thème du congrès où cette «petite poétique» a trouvé son premier public - à l'interaction, dans cette formule, de l'observation et de la narration, du spectacle et du récit, et à la manière dont ces deux registres s'agencent et s'articulent, tant au niveau de la progression narrative que sur le plan, plus concret, de leur cohabitation dans l'espace du volume.

Originellement publié en 1707, avant d'être profondément remanié en 1726, le Diable boiteux de Le Sage, inspiré de l'œuvre espagnole de «l'illustre» Luis Velez de Guevara, a pour héros un jeune bachelier madrilène, Cléofas Leandro Perez Zambullo, que divers déboires amoureux et nocturnes amènent à trouver refuge dans le galetas d'un

5 Didier Souiller, «Le récit picaresque», Littératures, 14, 1986, p. 13-26: «De même que surgissent dans le temps de nouveaux maitres, apparaissent de nouveaux personnages, selon un identique cheminement chronologique qui permet, avec ces derniers, un éclatement du récit, jusqu'alors centré sur l'autobiographe: rien n'interdit à celui qui survient de conter sa propre histoire. Les récits insérés sont une caractéristique de l'"esthétique" picaresque» (p. 14). 
puissant sorcier, en l'absence de ce dernier. Répondant à un appel à l'aide, dont la provenance le plonge d'abord dans une certaine perplexité, l'étudiant libère le diable Asmodée de la bouteille où le nécromant l'avait enfermé. Le diable, reconnaissant, venge Cléofas de celle qui l'a trahi, puis lui révèle les secrets de Madrid endormie: en soulevant les toits de la ville, Asmodée expose à son protégé diverses scènes, divers «spectacles», que son omniscience diabolique lui permet ensuite de commenter, de façon succincte ou avec force détails.

Ce canevas plutôt lâche - la narratophilie favorise les organisations simples, les structures accortes - est à même d'accueillir quatre différents modes de développement narratif. Il propose d'abord de courtes anecdotes, des saynètes moralisantes, des portraits rapidement brossés, qui forment la plus grande partie des "observations» de Cléofas et d'Asmodée. Ces morceaux se présentent en grappes, tantôt pêle-mêle (ch. III et IV), tantôt unifiés par un thème. Ces ensembles thématiques trouvent parfois leur principe commun dans un lieu, comme la prison (ch. VII), la maison des fous (ch. IX), l'église (ch. XII), la cour (ch. XVII), ou le monastère de la Merci (ch. XIX-XX) ${ }^{6}$; ailleurs, ils se consacrent à la réalisation systématique d'un programme satirique. Le chapitre VIII donne ainsi lieu à un contraste de l'activité nocturne des personnages observés avec la manière, évidemment contradictoire, dont ils ont occupé leurs journées. Au chapitre $X$, qui suit une visite aux «petites maisons», Asmodée porte à l'attention de Cléofas une série de "fous en liberté», qui mériteraient eux aussi l'enfermement. Le chapitre XVI est consacré aux songes, et le chapitre XVII aux occupations du petit matin.

6 Il n'est pas interdit de considérer la composition du Diable boiteux, dans son ensemble, comme une variation sur le schéma du voyage archipelagique, tel qu'on l'observe par exemple dans le Quart Livre, dans les Aventures de Télémaque ou dans le Nouveau Gulliver de Desfontaines (1730). Comme l'équipage de Pantagruel, comme Télémaque et Mentor, les héros de Le Sage se déplacent d'un îlot à un autre, auxquels correspondent les diverses unités du récit. La transposition de la formule ne se fait évidemment pas sans d'essentielles transformations: les îles de l'Atlantique ou de la Méditerranée deviennent les toits de Madrid, promontoires d'où le diable et le bachelier peuvent contempler la ville tout entière et en explorer les dessous; quant à l'élément «liquide», qui chez Rabelais ou Fénelon baignait l'espace interchapitral, il est ici remplacé par l'élément aérien, ce ciel nocturne que parcourt Asmodée. Ces transpositions merveilleuses exceptées, on retrouve chez Le Sage tous les traits structurels du modèle. On y remarque notamment cette correspondance de l'«île», de l'épisode et de l'unité de division - chapitre, livre ou partie - , qu'on peut désigner, en infléchissant la terminologie parfois absconse d'Éric Fougère, par le terme d'isoléité (les Voyages et l'ancrage. Représentation de l'espace insulaire à l'Age classique et aux Lumières (1615-1797), Paris, L'Harmattan, 1995, p. 12 et 232-233). 
Ces suites anecdotiques donnent aussi naissance à des développements narratifs plus étendus. On peut alors distinguer entre:

1. les nouvelles insérées, qui ne sont que l'amplification, la dilatation d'une anecdote, et se détachent par conséquent fort peu de l'inventaire litanique d'Asmodée ;

2. les nouvelles complètement autonomisées, soit $\mathrm{l}^{\prime}$ «Histoire du comte de Belflor» (ch. IV et V) et "La force de l'amitié» (ch. XIII et XV). La nouvelle est ici d'autant plus autonome et détachable qu'elle respecte la limite des bornes chapitrales, selon un principe de stricte équivalence entre chapitre et récit, que Le Sage respectera encore dans Guzman d'Alfarache (1732), dans les Aventures de Beauchesne (1732) et dans les premiers livres de Gil Blas. Chacune des nouvelles est introduite, ou réintroduite, par la prise de parole narratoriale d'Asmodée, qui correspond exactement au commencement du chapitre; dans trois chapitres sur quatre, la fin du segment métanarratif épouse également la (toute) fin du segment «physique». Isolées, singularisées de la sorte à l'intérieur de la séquence romanesque, les nouvelles ne sont cependant jamais complètement retranchées de leur situation interne d'énonciation. Chacune d'elles connaît une interruption, qui met à l'épreuve - et permet de confirmer - la coïncidence des deux systèmes de segmentation. L'«Histoire de Belflor» est ainsi brièvement suspendue par une question de Cléofas; l'échange qui s'ensuit n'a pas droit à un chapitre séparé, mais la reprise de parole d'Asmodée, elle, occasionne l'ouverture d'une nouvelle unité. La correspondance de la segmentation matérielle et de la répartition des niveaux narratifs est plus manifeste encore dans «La force de l'amitié», où l'échange intercalaire fait l'objet d'un chapitre complet, le quatorzième, assimilé par Asmodée à un simple excursus ${ }^{8}$.

7 C'est de ce premier type que relèvent par exemple, au chapitre VII, l'histoire de Guillaume, le garçon d'auberge déguisé en fantôme (333-340); au chapitre VIII, celle du bourgeois Patrice, trompé par deux aventurières (345-349); au chapitre IX, celle de Don Garcie, qui se travestit pour faire sa cour à sa maîtresse (354-358); ou encore, au chapitre XIX, celle de Fabricio, le fils de laboureur épris d'une aristocrate qui l'ignore (470-475). Ici comme plus loin, nous employons l'édition du Diable boiteux fournie par Étiemble dans le premier tome des Romanciers du XVIII siècle, Paris, Gallimard, coll. «Bibliothèque de la Pléiade», 1960.

8 «Il est temps, ajouta le boiteux, de finir la digression. Je vais reprendre le fil de l'histoire que vous avez interrompue» (ch. XIV in fine, 421). Ce statut digressif, posé 
Si elles sont d'abord dictées par la substitution des voix narratives, ces interruptions ne restent pourtant pas sans incidence sur l'économie «interne» des nouvelles ainsi saucissonnées. La coupure occasionnée par les remarques suspensives de Cléofas correspond, dans les récits eux-mêmes, à un point d'orgue, ou à un topos conventionnel de rupture: préparation de la confrontation de Cespedes et de Belflor, dont dépend l'avenir de Léonor, dans $l^{\prime}$ «Histoire de Belflor»; enlèvement de Théodora, dans «La force de l'amitié».

3. Le roman comprend enfin une nouvelle semi-autonomisée, l'histoire de Pablos de Bahabon, qui sans être isolée ou désignée de façon aussi univoque que les «véritables» nouvelles autonomisées, occupe la plus grande partie du chapitre XX, et se voit de surcroît attribuer un segment $\mathrm{d}^{\prime}$ intertitre, il est vrai purement allusif et rhématique («De la dernière histoire qu'Asmodée raconta...»). Respectant la procédure établie pour les nouvelles autonomisées, le chapitre commence avec la prise de parole métanarrative d'Asmodée; mais la nouvelle, si elle connaît une sorte de dénouement, n'est pas entièrement ou complètement conclue: la fin du chapitre est consacrée au «rappel» du diable dans le lieu de son supplice, qui interrompt sa péroraison et devient rétrospectivement le point focal du chapitre tout entier.

Chacun de ces récits, de la simple anecdote à la nouvelle autonomisée, prend source dans un «tableau» $(286,321,447)$, dans une «image» (285) ou dans une «scène» $(350,392)$, dont le caractère provisoirement incompréhensible rend nécessaire le commentaire diabolique. Le vocabulaire de la vision domine le roman. D'entrée de jeu, Asmodée promet à Cléofas de lui «montrer tout ce qui se passe dans Madrid» (277) et de lui fournir, par cette (dé)monstration, «une parfaite connaissance de la vie humaine» (278); le savoir passe ici par l'œil, par le regard, par le spectacle découvert

péremptoirement par Asmodée, est à tout le moins ambigu. L'anecdote relatée (la dispute du poète comique et du poète tragique) est un "hors d'œuvre», non par rapport au roman dans son ensemble (dont les anecdotes sont la matière, le propos même), mais par rapport à la nouvelle qu'elle interrompt - qui peut elle-même être perçue comme une digression «romanesque» dans la succession monotone des observations moralisantes. Cette ambiguïté est encore creusée par le dispositif, qui accorde un chapitre tant à l'épisode des poètes qu'à chacun des deux volets de «La force de l'amitié» - présentant donc les segments comme équivalents, et infirmant la (dis)qualification diabolique du chapitre XIV. 
et démystifié. Ce caractère «oculaire» de la connaissance est particulièrement marqué dans le cas des «simples» anecdotes, où c'est la scène elle-même qui constitue tout le récit: celui-ci se limite à une description détaillée, à laquelle Asmodée adjoint quelques commentaires généraux, suffisants pour déterminer le caractère et les relations des personnages observés.

L'élément visuel reste toutefois prégnant dans le cas des narrations plus développées. Les nouvelles insérées, autonomisées ou semi-autonomisées, sont toutes introduites selon une procédure figée. Il y a d'abord, comme dans l'anecdote, une perception, plus ou moins active, du tableau à expliquer. Tantôt, c'est le spectacle lui-même qui s'impose aux spectateurs, qui «attire [leurs] regards» (475) ou qui se "présente à [leur] vue» (280, 344); tantôt c'est Cléofas, toujours à l'affût, qui «remarque» une nouvelle scène $(323,415,475)$ - ou qui la «voit» $(360,389,456,459)$, l'«aperçoit» $(305,321,332,447,451,460,466)$, la «découvre» $(344,389)$, la "parcourt d'un œil curieux» (353). Le plus souvent, toutefois, l'initiative de la narration revient à Asmodée lui-même. Les injonctions du boiteux tapissent le roman: il ordonne à son disciple $\mathrm{d}^{\prime}$ «arrêter» son regard (286, $341,392)$ ou de le "laisser tomber» (321) sur une scène pittoresque; il lui suggère de «jeter» $(349,458)$ ou de «porter» $(323)$ la vue sur un objet surprenant; il l'enjoint de «voir» un spectacle intéressant $(285,373,451)$, ou de le «regarder» $(321,380,455)$, de l'«observer» $(323,326,382,455)$, de le «remarquer» $(285,391,467)$, de l'«examiner» $(333,362)$, de le «considérer» $(375,457,467)$, de le «découvrir» $(461)$, de lui «donner son attention» (323) - allant parfois jusqu'à le «montrer du doigt» (463). L'étonnement réel ou supposé du bachelier, devant la situation ainsi offerte à son regard, entraîne la prise de parole narrative du démon.

Le récit qui s'ensuit forme une boucle et se termine, après quelques pages ou plusieurs chapitres, par un retour au «présent» de l'énonciation démoniaque. Tous les éléments du récit convergent dans la scène, dans le spectacle initial, qui d'élément introductif et générateur devient épilogue et conclusion. On ne recense guère que deux exceptions à cette fermeture cyclique, à cette clôture de la nouvelle asmodéenne: le dernier récit inséré, celui de Fabricio (470); et la nouvelle «semi-autonome» mentionnée plus haut, l'histoire de Pablos de Bahabon. Dans le second cas, on l'a dit, un dénouement est au moins évoqué, suggéré par le récit; mais celui-ci ne parvient pas à compléter son parcours circulaire, à revenir au tableau initial. C'est qu'entre-temps l'instance narrative ellemême est disparue: rappelé dans sa bouteille, Asmodée ne peut remplir jusqu'au bout son travail d'élucidation. Loin de constituer une erreur, un oubli de la part de Le Sage - oubli d'autant plus improbable que notre homme, pour le meilleur et pour le pire, est l'un des plus sévères réviseurs de l'Ancien régime - , cette narration tronquée fait participer 
le lecteur au dépit diabolique. La liberté est refusée à Asmodée, mais c'est la fin de l'histoire, le fin mot du récit, qui sont niés au public, lequel devra se contenter de conclure lui-même l'aventure, à l'aide des indices qui lui ont déjà été livrés. Il s'agit d'une lacune "mimétique», qui induit aussi, paradoxalement, un certain «effet de réel» - dans la mesure où le méchant sorcier n'attend pas que le diable ait proprement bouclé son récit, pour lui faire connaître son châtiment; mais c'est une lacune sans grande importance, dans le système narratophilique du premier $\mathrm{XVIII}^{\mathrm{e}}$ siècle. L'inachèvement romanesque n'a pas encore ce caractère de scandale qu'il revêt pour nos yeux de modernes. Peu importe en somme qu'il y ait ou non clausule, que soient enfin noués tous les fils, tous les lacs et les entrelacs du récit: quelques personnages se sont agités, se sont aimés, se sont affrontés - c'est là tout ce que demande celui ou celle qui, en 1707 et 1726, s'est emparé du roman. Le destin en suspens de l'esclave racheté ne saurait (trop) indisposer un lecteur ou une lectrice qui, quelques années plus tard, envisagera avec résignation l'incomplétion définitive de la Vie de Marianne, du Paysan parvenu, des Égarements du cour et de l'esprit, et de la plus grande partie de l'œuvre romanesque de Mouhy.

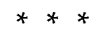

Dans la formule asmodéenne, un être magique révèle donc, au profit d'un personnage-témoin, les secrets d'une ville (diversement) endormie. Les observations s'accumulent, donnant lieu à de courts portraits satiriques, ainsi qu'à des passages narratifs plus développés. L'élément visuel revêt une importance primordiale: les scènes surprises servent à la fois de points de départ et d'arrivée, de déclencheur et de résolution. Enfin, le découpage chapitral, la disposition physique du roman, entre en congruence avec sa structure narrative ou épisodique. Le modèle ainsi instauré - ou, tout au moins, francisé - par Le Sage fera l'objet de nombreux investissements tout au long du XVIII siècle. Ces reprises prendront elles-mêmes plusieurs formes, de plus en plus éloignées du canevas et de l'univers d'origine.

Le Diable boiteux donne d'abord lieu à des suites en bonne et due forme, caractérisées par une reprise au moins partielle du personnel du romansource. Dans le Diable babillard ou indiscret, de De Campan (1711), c'est un nouveau démon qui entraîne Cléofas au-dessus de Madrid, dont il a à son tour le pouvoir de soulever les toits et de commenter les mœurs. Les chapitres tantôt correspondent à un épisode isolé (I, II, III), tantôt se regroupent, de manière à contenir une narration plus étendue (IX à XIV, XV à XIX). À l'inverse, dans le Nouveau Diable boiteux de Publicola Chaussard (1799), c'est le personnage d'Asmodée qui, à près d'un siècle 
d'intervalle, sert de lien avec le récit d'origine, en prenant sous sa tutelle un nouveau bachelier, dont il cherche à refroidir les ardeurs révolutionnaires". Le sous-titre retenu par Chaussard, "Tableau philosophique et moral de Paris», annonce immédiatement une transformation du projet asmodéen. Les anecdotes narratives que privilégiait un Le Sage, et que lui ont emprunté la plupart de ses épigones, restent présentes (voir, notamment, les chapitres VII à XV, ou XXVIII à XXXIV); mais elles laissent souvent la place à de longues suites discursives et théoriques sur les marchés (XXIV à XXV), les écoles (XXXV à XXXVII), les mœurs (XLII à LII), le commerce (LIX à LXV), les théâtres (LXXII à XC) 一, entrecoupées de courtes scènes exemplaires ou «démonstratives».

Plus éloignées encore du texte d'origine, les reprises formulaires se contentent d'adopter le modèle du roman asmodéen, en substituant au diable un nouveau personnage merveilleux et en remplaçant Madrid par une autre ville, exotique ou imaginaire. Ainsi les Spectacles nocturnes de Magny (1756) proposent-ils une série de petites histoires galantes, observées la nuit dans les maisons de "Cithéropolis», la ville des amours, nouveau masque du Paris des Lumières. Le narrateur est cette fois conduit par une fée, Almanzine, et cette sexualisation du guide surnaturel n'est pas sans conséquence. Le but des pérégrinations asmodéennes n'est plus strictement descriptif: le narrateur et la fée sont tous deux à la recherche de leurs amants respectifs, qu'ils trouveront, au petit jour, dans les bras l'un de l'autre. A l'inverse du Nouveau Diable boiteux, la structure narrative est ici plus «nouvellistique», moins anecdotique que dans le roman de Le Sage: chacun des vingt-cinq chapitres propose une histoire principale, parfois sertie de réflexions et d'observations moralisantes; quelques récits s'étendent sur plusieurs chapitres (voir, par exemple, $I^{\text {re }}$ partie, ch. II à VI, et VIII à IX).

Dans une autre transposition orientale de la formule asmodéenne, Une nuit de Babylone (anonyme, 1789), le jeune Hirzan et le génie Zariel, qu'un sortilège a rendu invisibles, observent la corruption des mœurs mésopotamiennes. Malgré un cadre encore manifestement irréaliste, tout élément burlesque a été ici évacué: la formule est traitée avec un sérieux inaltérable, que n'affichaient pas les performances antérieures.

9 Le roman s'inscrit dans une série d'ouvrages de la fin du XVIII ${ }^{\mathrm{e}}$ siècle qui revisitent les données de certains «classiques» de l'Ancien Régime, pour les confronter, avec des bonheurs divers, aux nouvelles réalités républicaines. Le jugement essentiellement négatif du Nouveau Diable boiteux correspond à ceux du Fils de Babouc à Persépolis (anonyme, 1790) ou du Voyage de M. Candide Fils au pays d'Eldorado (Bellin de la Liborlière, 1803). 
Comme pour les Spectacles nocturnes, les observations des deux comparses sont effectuées dans le cadre d'une enquête, purement intellectuelle cette fois, dont Zariel, en début de nuit, énonce l'enjeu:

Tu veux savoir si tu seras heureux en prenant une compagne, je vais te faire voir assez de femmes pour te déterminer: mais je ne peux fixer ton choix. Déjà la nuit tend ses voiles sur Babylone, voici le temps propre à découvrir la vérité. Nous allons nous promener sur la ville; nous verrons tout sans être vus, tes yeux pénétreront dans toutes les demeures, et dès qu'un objet piquera ta curiosité, je $t^{\prime}$ apprendrai ce que tu voudras savoir ${ }^{10}$.

Les vingt-cinq chapitres de ce sondage misogyne et panurgique contiennent tous une vignette ou une anecdote, observée d'abord de l'extérieur, puis expliquée par Zariel. Le romancier obtient à l'occasion un effet sériel, en faisant revenir certains personnages ou situations dans deux chapitres, à différents moments de la nuit: il crée ainsi une paire thématique avec les chapitres $V$ et $X X$, «Le souper» et "La suite du souper», puis avec les chapitres VI et XXII, «La femme fidelle» et «Le réveil de la femme fidelle» — qui l'est (évidemment?) moins qu'on ne le croyait.

On assiste en outre, dans la seconde moitié du siècle, à une sécularisation, à un singulier désenchantement de la formule. Le personnage fantastique est alors remplacé par un vulgaire mortel; les villes espagnoles ou orientales, qui servaient jusqu'alors d'alibi au microcosme parisien, abandonnent ces oripeaux fantaisistes; les conditions de l'observation prennent elles-mêmes un tour plus réaliste, dans lequel subsistent cependant certaines particularités «démoniaques». Cet asmodéisme désenchanté est préfiguré par un roman anonyme de 1772, les Matinées du Palais-Royal. Divisé en deux parties, sans toutefois être découpé en chapitres, l'ouvrage accumule les observations de deux amis, qui se retrouvent au Palais-Royal et commentent systématiquement l'attitude et le personnage des passants. Les «rôles» sont à nouveau clairement tranchés, entre un narrateur-annotateur et un interlocuteur «initié». La formule du roman fait l'objet, comme chez Le Sage ou Magny, d'un énoncé explicite, d'un véritable pacte inaugural:

Nous nous assimes pour jouir plus parfaitement du merveilleux tableau que nous offroit cette promenade. Il étoit d'autant plus intéressant pour nous que mon compagnon en connoissoit tous les objets. Il ne passoit devant nous per- 
sonne qu'il ne connût parfaitement, je n'avois qu'à lui témoigner la plus petite envie, il ne se faisoit pas prier pour m'en conter l'histoire $[\ldots]^{11}$.

La sécularisation de la formule est portée à son terme dans l'Optique du jour, ou le Foyer de Montansier (Rosny, 1799). Le diable et l'étudiant sont ici remplacés par deux jeunes élégants, qui observent en cachette les personnages, médiocres ou hautains, qui fréquentent le Théâtre des Variétés. Cette substitution mise à part, le schéma asmodéen est scrupuleusement repris: le vertueux d'Estival est posé en sévère Cléofas, alors que Floricourt - le léger, le libertin Floricourt — se voit sans ambiguïté arroger la fonction diabolique. Les termes dans lesquels il propose à son ami de l'accompagner au spectacle, après que d'Estival eût manifesté le désir de connaître les «ressorts», les «liens» et les «secrets» de la société, supposent une omniscience démoniaque, qui n'a rien à envier à celle d'Asmodée:

Je te conduirai ce soir au théâtre des Variétés, ci-devant Montansier; c'est là le rendez-vous des différentes classes de la société qui donnent le plus de prise contre elles, et sérieusement je suis à même de t'éclairer. Je connais à fond une grande partie des habitués de ce théâtre, et je puis te fournir les moyens de faire à leurs dépens de graves et sérieuses réflexions. Je ne te laisserai rien à désirer sur leur compte; je te ferai descendre jusque dans leurs ames; en un mot, je te ferai connaître jusqu'au moindre repli de leurs cœurs ${ }^{12}$.

Le roman est divisé en douze chapitres; chacun d'eux est consacré à un personnage, à un «type» différent, qui lui fournit à la fois son titre et son unité thématique ${ }^{13}$. Dans un ultime renversement de la relation asmodéenne, toutefois, c'est le «diable» Floricourt qui, à l'issue de ses observations, est converti au patriotisme et à la citoyenneté.

Il est enfin possible d'identifier certaines réalisations partielles ou dérivées de la formule. Giphantie de Thiphaigne de la Roche se présente ainsi comme un récit utopique "classique», répondant à la structure

11 Paris, Bastien, p. 19-21. Le texte a autrement la particularité d'articuler un double cadre, alors que les susdites observations du Palais-Royal (cadre 1) sont recueillies dans un manuscrit (cadre 2), que le narrateur lit ensuite à deux auditeurs.

12 Paris, Marchand, «Introduction», p. xi-xii; nos italiques.

13 On va ainsi de «L'agioteur» (ch. I) à «L'auteur» (XII), en passant par «Le rentier» (II), «La courtisanne» (III), «La fille publique» (IV), «Le nouveau parvenu» (V), «L'intriguant» (VI), «Le joueur» (VII), «L'anarchiste» (VIII), «L'émigré» (IX), «Le militaire» $(X)$ et «L'homme en place» $(X I)$. 
ternaire décrite par J.-M. Racault ${ }^{14}$; certains épisodes proposent cependant des micro-formules, dont le fonctionnement renvoie au modèle asmodéen. Dans l'épisode du «Globe», par exemple (I ${ }^{\mathrm{re}}$ partie, ch. VIII à $\mathrm{XI}$ ), une baguette et un globe magique permettent au narrateur de surprendre toute parole prononcée sur terre; chaque chapitre correspond dès lors au dialogue ou au soliloque surpris. On est plus proche encore du procédé du Diable boiteux dans l'épisode subséquent du «Miroir», aux chapitres VIII à XVII de la II ${ }^{\mathrm{e}}$ partie: employé en conjonction avec le globe et la baguette, le miroir permet non seulement d'entendre, mais aussi de regarder, de contempler la scène découverte «en temps réel». Des traces diffuses de la dynamique asmodéenne sont par ailleurs perceptibles jusque dans la «littérature du regard» de la fin du XVIII siècle. Nouveaux diables, le Mercier du Tableau de Paris et le Restif des Nuits de Paris entendent offrir un panorama complet, un portrait successif et exhaustif de la réalité parisienne. Malgré leurs différences de méthode, malgré leurs visions contradictoires de ce «réel» qu'ils ont pour charge de contenir et d'exprimer, ces ouvrages encyclopédiques, par leur caractère épisodique et cumulatif, rappellent la structure du roman asmodéen; un même dévoilement y a lieu, une même démystification, un même effort - explicite chez Mercier — de re-présenter au lecteur ce qui lui est devenu trop familier ${ }^{15}$. En «adaptant» la formule, Restif et Mercier procèdent à une fusion, à un télescopage des deux «rôles»: celui du maître omniscient, «surnaturel» ou démoniaque; celui de l'observateur étonné, auquel sont révélés les dessous, les secrets d'une cité qu'il croyait connaître. À moins qu'on n'attribue au narrateur-observateur la seule fonction diabolique et qu'on réserve au lecteur le rôle de l'étudiant, de l'apprenti, du néophyte. Une fois l'ouvrage terminé, une fois le narrateur appelé vers d'autres entreprises - comme le diable, rappelé dans la bouteille du sorcier - , le lecteur reste seul devant cette ville, cet univers à réformer. Dès la «Préface» du Tableau de Paris, tout le portrait de la capitale tend vers cette fin pragmatique; le lecteur, nouveau Cléofas, doit rentrer dans le monde - non pas, comme l'étudiant de Le Sage, pour

14 Voir Jean-Michel Racault, l'Utopie narrative en France et en Angleterre. 1675-1761, Studies on Voltaire and the Eighteenth Century, 1991, p. 462 et suivantes.

15 «Beaucoup de ses habitans sont comme étrangers dans leur propre ville: ce livre leur apprendra peut-être quelque chose, ou du moins leur remettra sous un point de vue plus net \& plus précis, des scenes qu'à force de les voir, ils n'appercevoient pour ainsi dire plus; car les objets que nous voyons tous les jours, ne sont pas ceux que nous connoissons le mieux» (Louis-Sébastien Mercier, Tableau de Paris, Amsterdam, 1782, "Préface», p. iv; Genève, Slatkine Reprints, 1979). 
épouser une jeune héritière et rejoindre cette société qu'il vient de surprendre sous son plus mauvais jour, mais pour agir, pour prendre à son compte la force décapante, la lucidité démoniaque du volume, et en appliquer la leçon.

Pour Pierre Chartier ${ }^{16}$, le diable boiteux est l'allégorie, l'incarnation même du roman des Lumières; il concentre en lui l'indiscrétion, l'impudeur, $l^{\prime}$ «infraction» qui fondent le conte moral ou le récit libertin. «Asmodée» devient ici une sorte de nom de code, le chiffre de tout ce qui dévoile, ce qui révèle, ce qui pénètre jusqu'aux réalités enfouies. La figure du démon de Le Sage prend une envergure spectaculaire; son indiscrétion est non seulement celle du personnage-monstrateur, mais aussi celle "du confident, de l'officieux ami, du tiers indispensable»; elle "s'étend à l'auteur et à l'éditeur du conte ${ }^{17}$ ", puis au lecteur même, ultime voyeur et divulgateur. Notre définition de l'asmodéisme est plus modeste: nous nous sommes concentré sur une formule, une structure caractérisée par une série de personnages, de types, de rôles récurrents, et par un certain mode d'agencement des niveaux narratifs. Nous n'avons pas cherché à faire de ce «roman asmodéen» une catégorie archétypale ou transhistorique; il s'agit au contraire d'un phénomène daté, qui s'inscrit dans une époque, dans un régime littéraire ou romanesque, et répond à certaines attentes, certains besoins de ce régime et de cette époque. L'asmodéisme, nous l'avons d'emblée précisé, n'est pas la seule de ces formules; aux noms de Mangogul, d'Amanzéi et de Gil Blas, évoqués en ouverture, on pourrait ajouter ceux de Candide, de Zadig, de Babouc, qui déterminent eux aussi un dispositif et commandent un certain nombre de suites, de reprises et d'adaptations. Il serait certainement fructueux de construire, à l'aide de ces formules, et de celles qu'elles recoupent ou côtoient, une histoire et une typologie de la fiction d'Ancien Régime. Il semble en tout cas y avoir là, au-dessus du topos, du motif et du thème, au-dessous du genre, du sous-genre ou de l'espèce, tout un champ de procédés, tout un niveau de phénomènes, jusqu'ici étrangement inexploré.

\section{UGO DIONNE}

Université de Montréal

16 Pierre Chartier, «Asmodée ou l'effraction», Dix-huitième siècle, 12, 1980, p. 209-218.

17 Ibid., p. 209. 